\title{
Criação de um Protótipo para Auxiliar no Processo de Desenvolvimento de Aplicativos Mobile para o Público Idoso
}

\author{
Fernanda Sipriano Marcilio \\ Universidade do Vale do Itajaí - \\ UNIVALI \\ fernandas.marcilio@edu.univali.br
}

\author{
Daniela Souza Moreira da \\ Silva \\ Universidade do Vale do Itajaí - \\ UNIVALI \\ daniela.moreira@univali.br
}

\author{
Anita Maria da Rocha \\ Fernandes \\ Universidade do Vale do Itajaí - \\ UNIVALI \\ anita.fernandes@univali.br
}

\begin{abstract}
With the growing number of elderly people in our society and the search of this population for technologies, more specifically the use of smartphones, and considering the physical and mental difficulties that aging causes to these people, there is a need to apply concepts of usability in mobile apps. However, there are technical and cultural barriers for the application of usability to be effective in meeting the needs of the elderly. Developing systems with agility as the market demands is antagonistic to the time required for application and usability evaluation in the software. Besides, the lack of usability requirements due to knowledge absence and time are some difficulties that the development team encounters in their daily lives. Given this context, the objective of this work was to create a prototype to help the development team to integrate usability, with a focus on elderly audiences, during the construction of a mobile application. To achieve this goal, the list of requirements, metrics and usability assessment methods focusing on mobile and elderly applications were selected from related works. Then the relationship between requirements, metrics, and assessment methods was identified and tested and the prototype was developed. Finally, with the analysis of the test it was possible to verify that the prototype reaches its objectives, as $88.9 \%$ of the participants would use the prototype in their projects and all stated that the prototype can contribute to the development of mobile applications.
\end{abstract}

\section{KEYWORDS}

Usabilidade, Idosos, Processo de desenvolvimento mobile.

\section{INTRODUÇÃO}

O número de idosos vêm crescendo consideravelmente. No Brasil, de acordo com o Instituto Brasileiro de Geografia e Estatística [IBGE 2018], estima-se que $32,17 \%$ da população terá mais de 60 anos em 2060. Além disso, o uso de celulares pelos idosos vêm aumentando cada vez mais. Em 2018, cerca de 69\% da população acima de 60 anos possuíam celulares e desta população aproximadamente $70 \%$ utilizavam para mandar mensagens, fotos, além de, ouvir música e acessar a internet [TELECO 2020]. Conforme os dados da Organização Mundial da Saúde [OMS 2015], o envelhecimento é um fator natural associado a danos biológicos e, com isto, ocorrem alterações nos sistemas sensoriais, tais como: as perdas de audição, visão e movimentos básicos. Por causa disso, essas dificuldades e alterações podem se tornar uma grande dificuldade para os idosos no uso da tecnologia no dia a dia [ANJOS e GONTIJO 2015]. A Organização das Nações Unidas [ONU 2020] cita que deve ser garantido aos idosos o benefício do uso das tecnologias sem ser tornar um desafio, pois ajudam os idosos a manter e fortalecer suas capacidades e a viver de maneira autônoma, independente e digna. Por isso, é importante aplicar usabilidade nas tecnologias, pois a usabilidade tem por objetivo a facilidade de uso e de aprendizado, memorização e produtividade na execução de tarefas, prevenção de erros e por último, satisfazer o usuário [BRASIL 2010]. Aplicar usabilidade nos sistemas é complexo e difícil de entender. Foi observado que existem três barreiras que impossibilitam a inserção de usabilidade nos sistemas: o tempo, pois o mercado exige desenvolvimento de sistemas em curto prazo de tempo e para pensar e aplicar em estratégias de usabilidade requer muito tempo; os requisitos, pois alguns sistemas não agregam os princípios de usabilidade nos seus requisitos; e a falta de conhecimento nos conceitos de usabilidade e tempo para estudar [ANJOS 2017]. Para isso, o objetivo deste trabalho foi criar um protótipo a fim de auxiliar a equipe a inserir com facilidade a usabilidade, com foco no idoso, durante o desenvolvimento de aplicativo móvel. Sendo que, um sistema com usabilidade focado para idoso, além de abranger maior número destes usuários, tende a ser mais aceito pelos usuários de outras faixas etárias, por motivo de que o aplicativo acaba sendo fácil de aprender, usar e memorizar. Além disso, reduz os custos e correções das funcionalidades do sistema e requer menos treinamento, suporte e manutenção durante o ciclo de vida [BRASIL 2010].

\section{FUNDAMENTAÇÃO TEÓRICA}

Neste Capítulo será abordado o conceito de idoso e suas dificuldades em relação ao uso das tecnologias. Logo após, é apresentado o conceito de usabilidade e seus métodos de avaliação. Por fim, é explicado sobre o processo de desenvolvimento de aplicativo mobile e suas atividades.

\subsection{Idosos}

A United Nations [UN 2015] define idosos pessoas com mais de 60 anos de idade, mas esse limite de idade pode variar de acordo 
com cada país. No Brasil, a lei $\mathrm{N}^{\circ} 10.741$ de $1^{\circ}$ de outubro de 2003 garante esse reconhecimento de pessoas idosas com mais de 60 anos [Brasil, 2003]. No entanto, a OMS (2005) defende que o envelhecimento não é marcado pela idade cronológica, e sim pelas variações relacionadas ao estado de saúde, participação e níveis de independências entre pessoas idosas.

Porém, a OMS (2015) afirma que o envelhecimento é um fator natural associado a danos moleculares e celulares e, por causa disso, ocorrem alterações nos sistemas sensoriais, tais como: as perdas de audição, visão e movimentos básicos. De acordo com Anjos e Gontijo (2015), tais alterações podem ocorrerem dificuldades em selecionar as informações sensoriais; na discriminação de sons, cores e percepção de fala; diminuição do equilíbrio, na acuidade visual, da noção de profundidade, na capacidade de adaptação ao claro e escuro, e dentre outras alterações. Além disso, de acordo com a OMS (2005), outras alterações que podem ocorrer em idosos são as perdas da capacidade cognitiva como a diminuição na velocidade de aprendizagem e na memorização.

Em função disso, "essas dificuldades e alterações podem se tornar um problema grave devido a inserção da tecnologia no dia a dia do idoso" [ANJOS e GONTIJO 2015]. Para isso, a ONU (2020) afirma que devem garantir que as pessoas idosas possam se beneficiar das tecnologias sem se tornar desafios no uso delas. Ainda, segundo a Organização, as tecnologias podem ajudar os idosos a manter ou fortalecer suas capacidades e que eles possam viver de maneira autônoma, independente e digna. Para isso, é crucial estudar e aplicar os conceitos de usabilidade nas tecnologias para que as habilidades físicas e cognitivas reduzidas pelo envelhecimento não afetem o uso destas tecnologias.

\subsection{Usabilidade}

A usabilidade é o estudo ou a aplicação de técnicas para prover facilidade de uso de um sistema [BRASIL 2010]. Ainda, de acordo com Brasil (2010), a usabilidade visa garantir que qualquer usuário consiga usar este sistema e que, também, funcione da forma esperada pelo usuário.

Além disso, a Associação Brasileira de Normas Técnicas [ABNT 2011] define que a usabilidade é o que um produto pode oferecer aos usuários específicos para realizar suas tarefas com eficácia, eficiência e satisfação de acordo com o contexto de uso específico. Sendo que eficácia é "[...] precisão e integridade com as quais os usuários alcançam os objetivos específicos [...]" [ABNT 2011]. A eficiência, de acordo com a ABNT (2011), são recursos utilizados para alcançar os objetivos, sendo que recursos incluem tempo, esforço humano, custos e materiais. Já a satisfação são "[...] medidas de respostas físicas, cognitivas e emocionais do usuário no uso do produto [...]" [ABNT 2011].

Ademais, o Brasil (2010) ainda cita que a usabilidade tem por objetivo a facilidade de uso e de aprendizado; memorização e produtividade na execução de tarefas; prevenção de erros; e por último, satisfazer o usuário. Para verificar isto, deve-se fazer avaliação de usabilidade, pois é utilizada para “[...] verificar o desempenho (eficácia e eficiência) da interação do usuário com o sistema e obter indícios do nível de satisfação dele [...]" [ANJOS 2017].

Além disso, para verificar se o sistema é eficaz, eficiente e possui satisfação do usuário, é aplicado métodos de avaliação de usabilidade. Anjos (2017) descreve alguns métodos de avaliação que são abordados a seguir:

1. Teste de usabilidade: Esta técnica consiste em realizar uma simulação e observar a interação do usuário com o sistema em um campo ou laboratório.

2. Percurso Cognitivo: Este método consiste em verificar os processos cognitivos dos usuários iniciantes através de lista de verificação orientada a tarefa.

3. Questionários e surveys: Este método tem por objetivo avaliar a satisfação do usuário em relação ao sistema.

A avaliação de usabilidade, além de verificar se o software atende os aspectos de eficácia e eficiência de modo que seja satisfatória para o usuário, apoia as equipes de desenvolvimento a coletar as métricas de usabilidade [ANJOS 2017].

As métricas de usabilidade têm por objetivo obter indicadores quantitativos e qualitativos relacionado a utilização de um sistema. Estes indicadores apontam sobre a experiência de usuário, através dos aspectos da eficácia, eficiência e grau de satisfação [ANJOS 2017]. Por isso, "Adquirir bons indicadores indica possivelmente que o sistema possui qualidade e conformidade com os requisitos" [ANJOS 2017].

Conclui-se que é importante que os sistemas atendam aos requisitos e às métricas de usabilidade para evitar problemas da falta de usabilidade. Além disso, para garantir que o sistema esteja atendendo esses requisitos e métricas, é importante aplicar "métodos de avaliação de usabilidade para identificar e diagnosticar problemas no sistema" [ANJOS 2017].

\subsection{Processo de desenvolvimento de aplicativo mobile}

Segundo Pressman e Maxim (2016), o aplicativo é um software voltado para plataforma móvel e aproveitam do mecanismo de interação disponíveis pela tecnologia e da interoperabilidade com recursos baseados na Web.

Porém, a complexidade para desenvolver softwares vêm crescendo e, além disso, os clientes estão exigindo cada vez mais a qualidade dos sistemas. Por isso é necessário entender a disciplina de engenharia de software, pois ela engloba um conjunto de métodos que auxiliam os profissionais a desenvolver um sistema de qualidade [PRESSMAN e MAXIM 2016].

Além disso, as plataformas móveis são complexas, pois além de possuir diferentes sistemas operacionais, também possuem diferentes tamanhos de telas, o que ocasiona em dificuldade de projetar a interface do usuário e diminuir a exibição dos conteúdos. Também, os aplicativos móveis podem facilmente ser interrompidos, ter oscilação da conectividade e limitação da bateria.

Por isso, para o desenvolvimento de aplicativos móveis, utilizamse ciclos de desenvolvimentos curtos, principalmente os processos de desenvolvimento ágeis e espiral. Pois é difícil prever como um 
aplicativo móvel vai evoluir com o tempo, visto que as circunstâncias do mercado são versáteis, além disso, os usuários têm suas necessidades alteradas constantemente e não conseguem definir por completo os requisitos do sistema [PRESSMAN e MAXIM 2016].

Além disso, deve-se adotar a qualidade de um aplicativo móvel durante todo o processo de desenvolvimento [PRESSMAN e MAXIM 2016] pois garante uma boa experiência do usuário nos aplicativos móveis.

\section{REVISÃO BIBLIOGRÁFICA}

Inicialmente, foi realizado um levantamento bibliográfico no qual foi possível identificar trabalhos que abordam temas semelhantes ao deste projeto para definir as funcionalidades e levantar requisitos. Para isto, a busca foi realizada entre março e maio de 2020 no ambiente Google Acadêmico e ACM Digital Library, limitando o período das publicações dos artigos entre 2015 e 2019. Foram utilizadas as palavras-chave: usabilidade, idoso, mobile e processo de desenvolvimento de software no ambiente Google Acadêmico; e usability, elderly OR older, mobile OR Smartphone e software development process no ambiente ACM Digital Library. Após aplicar os critérios de inclusão e exclusão, foram selecionados três trabalhos correlatos, que serão detalhados nas próximas seções.

\subsection{Verificação de usabilidade para smartphones com foco no usuário idoso}

Souza Filho (2019) visa propor soluções para melhoria da usabilidade em aplicativos mobile com foco em público idoso. A solução proposta pelo autor foi criar uma lista de verificação através dos estudos de observações dos idosos e das análises das heurísticas propostas por outros autores. Por fim, após identificar algumas heurísticas existentes na literatura, Souza Filho (2019) fez uma análise comparativa a fim de gerar uma lista de verificação com 43 questões para sistemas móveis focado em problemas específicos para idosos, no qual foi avaliado por especialistas na área de usabilidade.

\subsection{Integrando a usabilidade ao processo de desenvolvimento de sites e-commerce}

A tese de Anjos (2017) tem o foco no desenvolvimento de uma ferramenta web para auxiliar e apoiar a aplicação de usabilidade durante todo o processo de desenvolvimento de criação de sites ecommerce.

Pois, um dos problemas citado pela autora pela falta de características de usabilidade em websites é a grande quantidade de usuários que abandonam o carrinho de compra, e os motivos são causados pela navegação inadequada no site, dificuldade de encontrar o carrinho de compras, poucas informações e imagens sobre o produto e processo longo e confuso para fazer a compra de um produto.
Para tentar atingir os seus objetivos, primeiramente, a autora aplicou questionários para identificar as dificuldades que as equipes enfrentam para desenvolver aplicativos web com usabilidade e, além disso, tentou integrar os requisitos, métricas e métodos de avaliação de usabilidade no processo de desenvolvimento de software.

Para isso, Anjos (2017) identifica e relaciona requisitos e métricas de usabilidade. Pois, segundo a autora, este relacionamento facilita identificar os indicadores. Além disso, a autora relaciona as métricas com métodos de avaliação de usabilidade, pois garante que os requisitos estão sendo cumpridos através da identificação e análise das métricas de usabilidade coletadas durante a avaliação. Após a avaliação da ferramenta final, foi verificado que a ferramenta auxilia a equipe durante o processo de desenvolvimento de software, além disso, permitiu a equipe a ter melhor conhecimento sobre requisitos, métricas e métodos de avaliação de usabilidade. A autora conclui que a ferramenta pode ser usada como referência para desenvolvimento de aplicações web e-commerce.

\subsection{Recomendações de usabilidade e acessibilidade para interface de telefone celular visando o público idoso}

O trabalho de Anjos e Gontijo (2015) visa propor recomendações gerais de usabilidade e acessibilidade para aplicar na interface dos celulares com foco nos idosos. Um dos motivos para desenvolver as recomendações é a falta de trabalhos que abordam sobre usabilidade, celular e idosos simultaneamente.

Para isso, as autoras aplicaram três técnicas de avaliação de interface definidas a seguir:

1. Questionário de Satisfação: foi aplicado um questionário com 31 perguntas a fim de avaliar a interação dos idosos com o celular. Após a análise da avaliação, foram identificados funções e suas nomenclaturas que são mais compreensíveis para os usuários idosos.

2. Arranjo de Cartas: esta avaliação tem por objetivo entender as navegações, os menus e taxonomias da interface. Utilizando as funções identificadas na avaliação anterior, foram feitos dois tipos de cartões: um grupo de cartões principais, que tinham funções principais identificados na avaliação anterior, como por exemplo a função chamadas; e um grupo de cartões secundários com subfunções dos cartões principais, como atender chamadas. Após isso, o participante precisa relacionar os cartões secundários com os cartões principais.

3. Teste de Compreensão de Ícones: esta avaliação tem por objetivo verificar quais ícones relacionados a suas funções são fáceis de identificar pelo usuário. Nesse teste, o usuário tinha que relacionar ícones de acordo as funções identificadas na primeira avaliação.

Por fim, Anjos e Gontijo (2015) concluíram após as análises dos testes que foram identificados problemas que os idosos possuem 
ao interagir com tecnologias móveis, e com base nisso, foram desenvolvidas as recomendações de usabilidade e acessibilidade com o intuito de auxiliar a equipe de desenvolvimento na tomada de decisão para elaborar interfaces eficientes, eficaz e fácil de usar.

\subsection{Considerações}

A partir da análise comparativa das características apontadas na seção anterior entre os trabalhos selecionados, foi observado que todos contemplam a usabilidade como foco do trabalho e atingem seus objetivos propostos.

Porém, apenas um dos trabalhos selecionados disponibiliza uma ferramenta para facilitar a inserção de usabilidade durante o processo de desenvolvimento de software. Este trabalho, por sua vez, não possui foco em idosos e aplicativos móveis.

Por fim, foi analisado que o tema usabilidade, idosos e celular ainda são bastante frequentes nas pesquisas, porém ainda é um tema difícil de ser entendido e aplicado na prática.

\section{DESENVOLVIMENTO}

O protótipo desenvolvido tem como objetivo ser utilizado pela equipe de desenvolvimento para auxiliar e orientar na criação de aplicativos móveis com qualidade e que inclua maior número de usuários. O protótipo auxilia no gerenciamento e avaliação dos requisitos de usabilidade com foco nos idosos.

Além disso, o protótipo foi desenvolvido no ambiente web utilizando React e Node.js. O React é uma biblioteca que utiliza a linguagem de programação JavaScript para construção da interface e utiliza o conceito de Single Page Application (SPA), ou seja, aplicativo de página única, garantindo performance para o usuário. Já o Node.js é um ambiente de execução que utiliza o JavaScript no lado do servidor, isso garantiu maior produtividade por utilizar a mesma linguagem de programação na aplicação e no servidor.

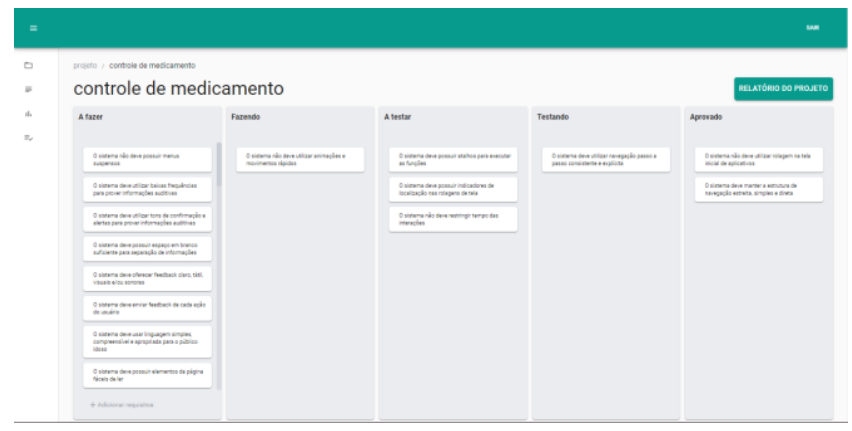

Figura 1: Tela do protótipo de visualizar o projeto.

O protótipo apresenta os requisitos, métricas e avaliação de usabilidade para aplicativos móveis e idosos. Com isso, o usuário pode gerenciar os requisitos de usabilidade através do kanban com as seguintes colunas: A fazer, Fazendo, A testar, Testando e Aprovado como mostrado na figura 1. Para o usuário avaliar se os requisitos estão sendo aplicados corretamente no seu aplicativo móvel, o protótipo aponta as métricas de usabilidade que deveriam obter para medir os requisitos e os métodos avaliações de usabilidade que podem obter as determinadas métricas.

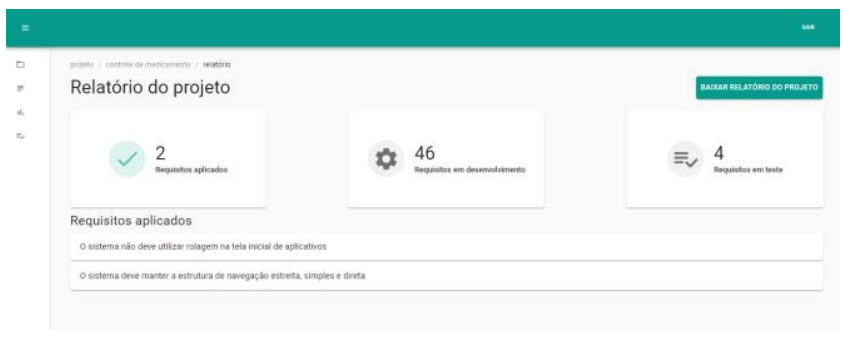

Figura 2: Tela do protótipo de relatório do projeto.

Além disso, o protótipo permite que o usuário gerencie os requisitos, métricas e métodos de avaliação, através da exclusão, inserção ou atualização e permite visualizar e gerar o relatório dos status dos requisitos do projeto em desenvolvimento como mostrado na figura 2 .

\section{DESCRIÇÃO DOS EXPERIMENTOS}

Nesta seção será apresentado o detalhamento dos experimentos. Foram realizados dois testes:

1. Validar a lista de requisitos, métricas e métodos de avaliação de usabilidade;

2. Verificar se o sistema desenvolvido atinge o objetivo específico deste trabalho.

Em ambos os testes, foram aplicados questionários e selecionados grupos de voluntários da área de computação. Para estes testes foram apresentados os resultados qualitativos dos dados.

No primeiro teste seis (6) participantes responderam remotamente um formulário com 69 questões. Ele foi dividido em três partes:

1. Análise de requisitos de usabilidade: consiste em verificar se os requisitos apresentados no teste consistem como requisitos de usabilidade.

2. Análise de métricas de usabilidade: o objetivo é verificar quais métricas é possível medir os requisitos de usabilidade apresentado nas questões.

3. Análise de métodos de avaliação de usabilidade: tem por objetivo identificar quais métodos de avaliação de usabilidade obtém-se cada métrica de usabilidade.

No questionário referente a análise de requisitos e métricas de usabilidade foi utilizada escala likert. Para cada pergunta há cinco (5) opções de respostas, uma escala que vai de 1 a 5, onde o "1" significa que "discorda totalmente", "2" é "discorda parcialmente", "3" é "Não concorda e não discorda", "4" é "concorda parcialmente" e no " 5 " é "concorda totalmente". Para identificar possíveis oportunidades de melhoria na lista de requisitos e métricas foi orientado aos participantes que justificassem a resposta caso a opção selecionada fosse entre $1 \mathrm{e}$ 3. 
Já na parte de análise de método de avaliação de usabilidade foram apresentadas questões de múltiplas escolhas com a lista de método de avaliação de usabilidade para cada métrica de usabilidade identificada neste trabalho. Porém o método de avaliação de usabilidade "Arranjo de cartas" e "Entrevista" não foram inseridos no questionário por conta de o questionário estar muito extenso e iria ficar muito cansativo para o voluntário.

Do segundo teste participaram nove (9) pessoas e foi realizado remotamente um teste de observação do sistema e no final foi aplicado questionário. $\mathrm{O}$ teste de observação foi realizado utilizando o software TeamViewer, o qual permite o acesso remoto e compartilhamento de área de trabalho e é compatível com muitos sistemas operacionais, como: Windows, Linux, Android, OS X, entre outros. O voluntário acessava o sistema através do computador do avaliador, realizava as tarefas propostas e, com isto, o avaliador conseguia observar e anotar pontos críticos no sistema. As tarefas foram colocadas junto ao questionário e o teste foi constituído por sete (7) tarefas relacionadas ao fluxo principal do sistema: desde entrar no sistema, gerenciar os requisitos no kanban e visualizar o relatório.

\section{RESULTADOS}

Nesta seção será detalhado os resultados obtidos dos testes. $\mathrm{Na}$ seção 6.1 será apresentado a análise obtido do teste de requisitos, métricas e métodos de avaliação de usabilidade. Já na seção 6.2, será apresentado os resultados apresentados no teste do sistema

\subsection{Resultado do teste de requisitos, métricas e métodos de avaliação de usabilidade}

$\mathrm{Na}$ parte dos resultados dos requisitos, apenas 1 resposta foi respondida como neutra, enquanto 41 foram respostas positivas. De acordo com a análise do teste, os requisitos proporcionam para que um sistema possua feedback imediato, tenha ações mínimas e pouca densidade informacional.

Além disso, no final do questionário sobre os requisitos, foram comentadas algumas observações, um deles era sobre uma sugestão de reduzir a densidade de informação, no qual foi comentado: "Uma forma de reduzir a densidade de informação da opção para configuração de adaptabilidade e acessibilidade, seria induzir o usuário a configurar essas opções inicialmente antes de utilizar a aplicação, não tendo a necessidade de deixar sempre aparente essas configurações. Caso haja a necessidade de readaptar, reiniciar essas telas de configuração. Exemplo parecido seria as configurações dos televisores que temos hoje.", com isso, foi acrescentado mais dois requisitos: "O sistema deve apresentar opções de configuração de adaptabilidade e acessibilidade, ao iniciar a aplicação pela primeira vez" e "O sistema deve permitir modificar ou reiniciar as opções de configuração de adaptabilidade e acessibilidade".

Já em relação às métricas, aproximadamente duas questões foram apontadas como discordo parcialmente ou totalmente, seta foram respondidos como neutras (não concorda e nem discorda), por fim, 159 das respostas foram satisfatórias.
Baseado nas respostas e observações dado pelos voluntários algumas métricas foram modificadas, tais como: a métricas "Número médio de mídia de áudio por página" foi trocada por "Média da frequência de som utilizado no sistema", pois de acordo com o comentário: "Número de mídia não diz respeito à frequência utilizada nos áudios, e como propõem nos requisitos uma frequência baixa, essa métrica não é capaz de distinguir isso".

Além disso, foram inseridas novas métricas com base nas análises da pesquisa. Como por exemplo, para a métrica "O número de erros do usuário" foi feito sugestão de requisito relacionado a métrica, no qual foi adicionado ao projeto: "O sistema deve salvar as configurações ou as ações realizadas pelo usuário".

Para a análise dos métodos de avaliação de usabilidade, para cada métrica tinha opções de múltiplas escolhas para os métodos de avaliação, foram aprovadas para o projeto todos que tinham pontos igual ou superior a três. Para pontos entre 1 e 2 , foram feitas algumas análises para verificar se pela métrica dava para medir através do método de avaliação de usabilidade.

Por fim, conclui-se que o resultado do teste foi satisfatório e poucos ajustes foram necessários na lista de requisitos, métricas e métodos de avaliação de usabilidade e suas relações.

\subsection{Resultado do teste do sistema}

A análise do teste do sistema foi realizada em duas etapas: análise do questionário e análise da anotação exercido durante o teste de observação. Em relação ao questionário, foi respondido por nove (9) participantes, sendo a maioria composta por desenvolvedores e estudantes da área da computação, no qual é mostrado na figura 3.

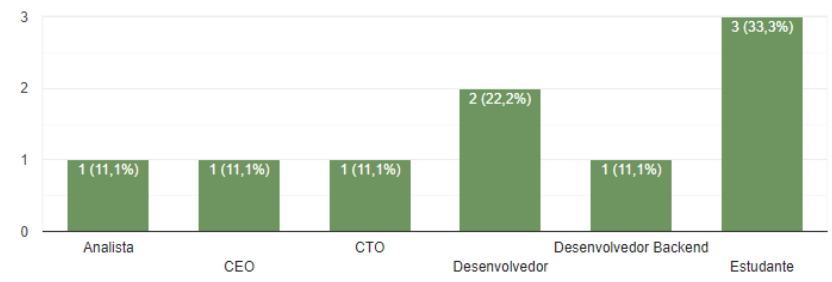

Figura 3: Gráfico do resultado do cargo dos participantes.

Já em relação ao nível de conhecimento em usabilidade dos participantes, grande parte dos participantes possuíam conhecimento intermediário em usabilidade, como mostrado na Figura 4. 


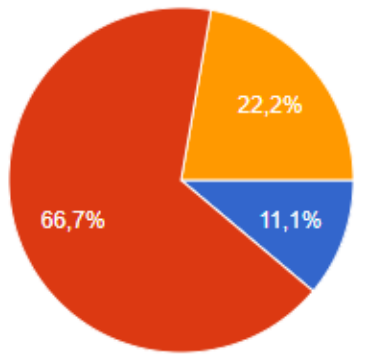

Básico Intermediário Avançado

Figura 4: Gráfico do resultado de conhecimento em usabilidade dos participantes.

Além disso, seis participantes já trabalharam com desenvolvimento de aplicativos móveis, porém apenas dois participantes trabalharam com desenvolvimento do sistema mobile aplicado e planejado com usabilidade e/ou com foco em idosos. Ainda assim, todos concordam que é importante inserir usabilidade com foco em idosos nos aplicativos, entretanto teve o seguinte comentário: "Mais ou menos, pois os idosos ficaram cada vez mais capacitados, entretendo acho que sempre haverá benefícios", por mais que os idosos estão mais capacitados, sempre haverá os limites físicos e cognitivos que ocorrem no envelhecimento e ao inserir a usabilidade acaba diminuindo o conflito entre os limites e o uso do aplicativo.

Por fim, oito participantes responderam que usaria o protótipo no seu projeto e um respondeu que não usaria por achar Trello similar e mais estabelecido, porém o Trello é um aplicativo de gerenciamento de projeto em geral e protótipo já predispõe os requisitos, métricas e métodos de avaliação de usabilidade para que o usuário gerencie os requisitos no projeto sem necessitar ter conhecimento em usabilidade. Em relação a isso, todos acreditam que o protótipo pode contribuir com os desenvolvedores de aplicativos móveis. No que se refere a indicar o protótipo para outros desenvolvedores, sete participantes responderam que indicariam e dois responderam que não indicariam pois "ainda está em um estágio muito inicial para que eu recomende".

Quanto a análise das anotações realizada durante o teste observação, foram observadas possíveis melhorias no protótipo. $\mathrm{O}$ teste de observação demorou em torno de 20 minutos para cada participante, sendo 3 minutos para explicar sobre o teste e o projeto, em média de 8 minutos para realizar as tarefas e 9 minutos para responder o questionário. $\mathrm{O}$ primeiro ponto observado foi que 4 voluntários demoraram alguns segundos para encontrar o botão de adicionar os requisitos na tela de kanban, alguns comentaram durante o teste que demoraram para perceber e/ou que perceberam na hora, mas ao voltar para tela de kanban novamente, esqueceram onde ficava o botão. Outro ponto foi na página de relatório, no qual demorou para perceber que mudava os requisitos ao clicar nas cards de "requisitos aplicados, "requisitos em andamento" e "requisitos completados". Além disso, a maioria comentou sobre a falta do modal de confirmação ao excluir algum item.

Durante o teste de observação, também foi comentado melhorias para nomes de alguns botões, ajustar alguns ícones e a possibilidade de editar e visualizar as informações da card no próprio kanban. Também, no teste obteve-se comentários positivos, como: o design do sistema é padrão e bonito, o sistema é intuitivo e possui os padrões visuais da web.

De maneira geral, conclui-se que o resultado do teste do protótipo foi positivo e ele atinge o objetivo geral proposto no trabalho.

\section{CONCLUSÃO}

Para iniciar este projeto, foi necessário realizar um estudo sobre usabilidade, idosos e processo de desenvolvimento de aplicativos móveis. Além disso, no decorrer da pesquisa foram identificadas as dificuldades que os idosos têm ao utilizar aplicativos móveis, pois muitos aplicativos não auxiliam nos problemas que os idosos possuem por conta do envelhecimento. Ainda nesta etapa, foram identificadas as dificuldades que a equipe de desenvolvimento tem para aplicar usabilidade durante o desenvolvimento de software. Por causa disto, foi proposta uma solução para minimizar os problemas de aplicar usabilidade em aplicativos móveis e com isso melhorar a qualidade do aplicativo e incluir o público idoso. Para propor esta solução foi identificado relação entre requisitos e métricas, pois com a métrica é possível avaliar se os requisitos estão implementados de maneira correta. Também foi identificado relação entre métricas e métodos de avaliação de usabilidade, já que através dos métodos de avaliação de usabilidade que se obtém as métricas. Em seguida, foi realizado um teste com o objetivo de verificar se os requisitos, métricas, métodos de avaliação de usabilidade e suas relações identificadas estão de acordo com o objetivo proposto.

Logo depois, foi desenvolvido o protótipo com o objetivo de ser um gerenciador de requisitos de usabilidade, com foco em idosos, para serem inseridos em aplicativos móveis.

Por fim, foi feito o teste do protótipo, onde foi analisado que $88,9 \%$ dos participantes usariam o protótipo e todos acreditam que o protótipo pode contribuir com os desenvolvedores de aplicativos móveis. Ainda no resultado do teste do protótipo obteve-se comentários positivos dos participantes como: "O design do sistema é padrão e bonito, o sistema é intuitivo e possui os padrões visuais da web" e "O que me chamou mais atenção foi o recurso de gerar relatório, porque com ele fica mais fácil ter um controle para se utilizar em uma reunião [...]”. Com isto, concluise que o protótipo atinge seu objetivo geral e que será capaz de auxiliar os desenvolvedores a integrar usabilidade, com foco em idosos, durante o desenvolvimento de seu aplicativo móvel.

\section{AGRADECIMENTOS}

Os autores agradecem a todos os voluntários que participaram desta pesquisa. 
XII Computer on the Beach

7 a 9 de abril de 2021, Online, SC, Brasil.

Marcilio et al.

\section{REFERÊNCIAS}

[1] Instituto Brasileiro De Geografia e Estatística (IBGE). Projeções da População do Brasil e Unidades da Federação por sexo e idade: 2010-2060. 2018.

[2] Teleco, teleco (2020). Estatísticas de Celulares no Brasil. Estatísticas de usuários de celulares no Brasil. Disponível em https://www.teleco.com.br/ncel usu.asp. Acessado em 09 abr 2020.

[3] Organização Mundial da Saúde (OMS). Relatório mundial de envelhecimento e saúde. Estados Unidos, v. 30, p. 12, 2015.

[4] ANJOS, Thaiana Pereira dos; GONTIJO, Leila Amaral. Recomendações de usabilidade e acessibilidade para interface de telefone celular visando o público idoso. Production, v. 25, n. 4, p. 791-811, 2015.

[5] Organização das Nações Unidas (ONU). A ONU e as Pessoas Idosas. Disponível em https://nacoesunidas.org/acao/pessoas-idosas/. Acessado em 20 mar 2020.

[6] Brasil. Ministério do Planejamento, Orçamento e Gestão. Secretaria de Logística e Tecnologia da Informação. Padrões Web em Governo Eletrônico: Cartilha de Usabilidade / Ministério do Planejamento, Orçamento e Gestão, Secretaria de Logística e Tecnologia da Informação - Brasília: MP, SLTI, 2010.

[7] ANJOS, Thaiana Pereira dos. Integrando a usabilidade ao processo de desenvolvimento de sites e-commerce: criação de ferramenta com foco nos requisitos, métricas e métodos de avaliação da usabilidade. 2017. 449 f. Tese (Doutorado) - Curso de Engenharia da Produção, Universidade Federal de Santa Catarina, Florianópolis, 2017.

[8] United Nations (UN). Department of Economic and Social Affairs; Population Division. World Population Ageing 2015. 2015.

[9] Brasil. Lei $n^{\circ} 10.741$, de $1^{\circ}$ de outubro de 2003. Dispõe sobre o Estatuto do Idoso e dá outras providências. Diário Oficial da União, p. 1-1, 2003.

[10] Organização Mundial da Saúde (OMS). Envelhecimento ativo: uma política de saúde. Organização Pan-Americana da Saúde-OPAS, 2005.

[11] Associação Brasileira de Normas Técnicas (ABNT). NBR ISO 9241-11: requisitos ergonômicos para o trabalho com dispositivos de interação visual parte 11: orientações sobre usabilidade. Rio de Janeiro, 2011.

[12] Pressman, Roger S.; Maxim, Bruce R.; Engenharia de software: uma abordagem profissional. 8. ed. Porto Alegre: AMGH, 2016.

[13] Souza Filho, Marcos Roberto Tenório de. Validação das estratégias de verificação de usabilidade para smartphones com foco no usuário idoso. 2019. 193 f. Dissertação (Mestrado) - Curso de Design, Universidade Federal de Pernambuco, Recife, 2019. 